\title{
Computing the output distribution and selection probabilities of a stack filter from the DNF of its positive Boolean function
}

\author{
Marcel Wild \\ Department of Mathematical Sciences, University of Stellenbosch \\ Private Bag X1, Matieland 7602, South Africa
}

\begin{abstract}
Many nonlinear filters used in practise are stack filters. An algorithm is presented which calculates the output distribution of an arbitrary stack filter $S$ from the disjunctive normal form (DNF) of its underlying positive Boolean function (PBF). Our algorithm avoids to enumerate the models of the PBF one by one, and thus is considerably more efficient than previous methods. The so called rank selection probabilities can be computed along the way.
\end{abstract}

\section{Introduction}

Stack filters were invented in 1986 and have been a key topic of research in nonlinear signal processing ever since. Simply put, all aspects of a stack filter are reflected in its underlying positive Boolean function, and a basic familiarity of the latter concept is all that is required to understand this article. Using Google Scholar one can easily track the literature on various other aspects of stack filters, e.g. their output distribution. In this article we present a new algorithm to calculate the output distribution. The new method, called stack filter $n$-algorithm, is an extension of the noncover $n$-algorithm [13] which generates, in compact form, all noncovers $X$ of given sets $A_{1}^{*}, \ldots, A_{h}^{*}$ (i.e. $X \nsupseteq A_{i}^{*}$ for all $1 \leq i \leq h$ ).

The stack filter $n$-algorithm is introduced by means of a medium-size example in Section 2 . Section 3 is dedicated to its theoretic assessment. Section 4 touches upon five related matters, among which a numeric evaluation, and extensions of the stack filter $n$-algorithm that deliver the telling selection probabilities of [8], respectively handle the balanced stack filters of [12].

\section{The stack filter $n$-algorithm}

Fix $m \geq 1$ and put $w:=2 m+1$. Let $b:\{0,1\}^{w} \rightarrow\{0,1\}$ be a positive Boolean function (PBF), i.e. one without negated variables. Refering to e.g. [2], an operator $S$ from $\mathbb{R}^{\mathbb{Z}}$ in itself defined by the $k$-th component of $S z$ being

$$
[S z]_{k}:=b\left(z_{k-m}, \ldots, z_{k}, \ldots, z_{k+m}\right) \quad(k \in \mathbb{Z})
$$


is called a stack filter of window size $w$ based on $b$. Notice that the PBF $b$ in (1) has been extended from $\{0,1\}^{w} \rightarrow\{0,1\}$ to $\mathbb{R}^{w} \rightarrow \mathbb{R}$ in the usual way, i.e. by replacing the logical connectives $\wedge$ and $\vee$ by the minimum respectively maximum operation for pairs of real numbers (while keeping the symbols). So, if

$$
b\left(x_{-1}, x_{0}, x_{1}\right):=\left(\left(x_{0} \vee x_{1}\right) \wedge x_{-1}\right) \vee x_{0} \quad\left(x_{i} \in\{0,1\}\right),
$$

then

$$
b(3,2,4)=((2 \vee 4) \wedge 3) \vee 2=(4 \wedge 3) \vee 2=3 \vee 2=3 .
$$

By construction each stack filter $S$ is translation invariant in the sense that pushing the series $x$ ten units to the right and then applying $S$ yields the same as first applying $S$ and then pushing ten units to the right. So $S$ is completely determined by formula (1) for $k=0$.

Let $Z=\left(\ldots, Z_{-1}, Z_{0}, Z_{1}, \ldots\right)$ be a doubly infinite sequence of independent indentically distributed (i.i.d.) random variables. Let $F_{Z}(t)$ be their common (cumulative) distribution function, i.e. $F_{Z}(t):=\operatorname{Prob}\left(Z_{i} \leq t\right)$ is the probability that $Z_{i}$ is at most $t$. By translation invariance the output distribution $F_{S Z}(t):=\operatorname{Prob}\left((S Z)_{i} \leq t\right)$ is independent of $i$. It is known that there is a well defined function $\phi_{S}(p)$, called the distribution transfer of $S$, such that

$$
F_{S Z}(t)=\phi_{S}\left(F_{Z}(t)\right) \quad(t \in \mathbb{R}) .
$$

What's more, $\phi_{S}(p)$ is a polynomial which can be calculated [15], [2, p.223 ] as

$$
\phi_{S}(p)=\sum_{b(x)=0} p^{|\operatorname{Zero}(x)|} \cdot q^{|\mathrm{One}(x)|}
$$

where $q:=1-p$ and $b$ is as in (1). The summation is over all bitstrings $x \in\{0,1\}^{w}$ with $b(x)=0$, where by definition

$$
\begin{aligned}
& \operatorname{Zero}(x):=\left\{1 \leq i \leq w \mid x_{i}=0\right\} \\
& \operatorname{One}(x):=\left\{1 \leq i \leq w \mid x_{i}=1\right\} .
\end{aligned}
$$

For instance, consider this positive Boolean function $b_{1}$ which is already in disjunctive normal form (DNF). It is of type $\{0,1\}^{9} \rightarrow\{0,1\}$ but we like to scale as $\{0,1\}^{W} \rightarrow\{0,1\}$ with $W:=\{-4,-3,-2,-1,0,1,2,3,4\}$ :

$$
\begin{aligned}
b_{1}\left(x_{-4}, \ldots, x_{4}\right) & =\left(x_{-2} \wedge x_{-1} \wedge x_{0}\right) \vee\left(x_{-1} \wedge x_{0} \wedge x_{1}\right) \vee\left(x_{0} \wedge x_{1} \wedge x_{2}\right) \\
& \vee \quad\left(x_{-4} \wedge x_{-3} \wedge x_{-2} \wedge x_{1} \wedge x_{2} \wedge x_{3}\right) \vee\left(x_{-3} \wedge x_{-2} \wedge x_{-1} \wedge x_{1} \wedge x_{2} \wedge x_{3}\right) \\
& \vee\left(x_{-3} \wedge x_{-2} \wedge x_{-1} \wedge x_{2} \wedge x_{3} \wedge x_{4}\right)
\end{aligned}
$$

In view of (2) we wish to encode the family Mod of all $x=\left(x_{-4}, x_{-3}, \ldots, x_{4}\right)$ in $\{0,1\}^{W}$ with $b(x)=0$ in a compact way*. First note that

$$
\operatorname{Mod}=\operatorname{Mod}_{1} \cap \operatorname{Mod}_{2} \cap \operatorname{Mod}_{3} \cap \operatorname{Mod}_{4} \cap \operatorname{Mod}_{5} \cap \operatorname{Mod}_{6},
$$

\footnotetext{
*If we were to start with the conjunctive normal form (CNF) of $b$, we would end up with a compact representation of the set $\operatorname{Mod}^{\prime}$ of all $x \in\{0,1\}^{W}$ with $b(x)=1$. Hence, instead of (2), a dual kind of formula would yield $\phi_{S}(p)$.
} 
where the family $\operatorname{Mod}_{i}$ corresponds to the $i$-th conjunction in (3). For instance we write

$$
\operatorname{Mod}_{1}:=\left\{x \in\{0,1\}^{W} \mid x_{-2} \wedge x_{-1} \wedge x_{0}=0\right\} \quad=\quad(2,2, n, n, n, 2,2,2,2)
$$

because $x_{-2} \wedge x_{-1} \wedge x_{0}=0$ (nul) if and only if at least one of $x_{-2}, x_{-1}, x_{0}$ is nul, and the other variables $x_{-4}, x_{-3}, x_{1}, x_{2}, x_{3}, x_{4}$ can independently assume the $\mathbf{2}$ values 0 and 1 . Thus $(1,1,0,1,0,1,0,1,1) \in \operatorname{Mod}_{1}$ but $(0,0,1,1,1,0,0,1,0) \notin \operatorname{Mod}_{1}$. If we identify a 0,1 -string $x$ with the subset $X=\left\{i \in W: x_{i}=1\right\}$ of $W$ then $\operatorname{Mod}_{1}$ consists of all noncovers $X$ of $A_{1}^{*}:=\{-2,-1,0\}$ in the sense that $X \nsupseteq A_{1}^{*}$. The noncover $n$-algorithm from [13] (more on that in Section 3) generates all simultaneous noncovers of the given sets (here deriving from the terms of a $\mathrm{PBF}) A_{1}^{*}, A_{2}^{*}, \ldots, A_{6}^{*}$ as follows:

\begin{tabular}{|c|c|c|c|c|c|c|c|c|c}
-4 & -3 & -2 & -1 & 0 & 1 & 2 & 3 & 4 & \\
\hline 2 & 2 & $n$ & $n$ & $n$ & 2 & 2 & 2 & 2 & $P C=2$ \\
\hline
\end{tabular}

\begin{tabular}{|l|l|l|l|l|l|l|l|l|l}
\hline 2 & 2 & 2 & $\mathbf{n}$ & $\mathbf{n}$ & 2 & 2 & 2 & 2 & $P C=3$ \\
\hline 2 & 2 & 0 & $\mathbf{1}$ & $\mathbf{1}$ & 0 & 2 & 2 & 2 & $P C=3$ \\
\hline
\end{tabular}

\begin{tabular}{|l|l|l|l|l|l|l|l|l|l}
\hline 2 & 2 & 2 & 2 & $\mathbf{0}$ & 2 & 2 & 2 & 2 & $P C=4$ \\
\hline 2 & 2 & 2 & 0 & $\mathbf{1}$ & $n$ & $n$ & 2 & 2 & $P C=4$ \\
\hline 2 & 2 & 0 & 1 & 1 & 0 & 2 & 2 & 2 & $P C=3$ \\
\hline
\end{tabular}

\begin{tabular}{|l|l|l|l|l|l|l|l|l|l}
\hline$n$ & $n$ & $n$ & 2 & 0 & $n$ & $n$ & $n$ & 2 & $P C=5$ \\
\hline 2 & 2 & 2 & 0 & 1 & $n$ & $n$ & 2 & 2 & $P C=4$ \\
\hline 2 & 2 & 0 & 1 & 1 & 0 & 2 & 2 & 2 & $P C=3$ \\
\hline
\end{tabular}

\begin{tabular}{|c|c|c|c|c|c|c|c|c|c}
\hline 2 & $\mathbf{n}$ & $\mathbf{n}$ & 2 & 0 & $\mathbf{n}$ & $\mathbf{n}$ & $\mathbf{n}$ & 2 & $P C=6$ \\
\hline 0 & $\mathbf{1}$ & $\mathbf{1}$ & 0 & 0 & $\mathbf{1}$ & $\mathbf{1}$ & $\mathbf{1}$ & 2 & $P C=6$ \\
\hline 2 & 2 & 2 & 0 & 1 & $n$ & $n$ & 2 & 2 & $P C=4$ \\
\hline 2 & 2 & 0 & 1 & 1 & 0 & 2 & 2 & 2 & $P C=3$ \\
\hline
\end{tabular}

\begin{tabular}{|l|l|l|l|l|l|l|l|l|l}
\hline 2 & $\mathbf{n}$ & $\mathbf{n}$ & 2 & 0 & 2 & $\mathbf{n}$ & $\mathbf{n}$ & 2 & final \\
\hline 2 & $\mathbf{1}$ & $\mathbf{1}$ & $\mathrm{n}$ & 0 & 0 & $\mathbf{1}$ & $\mathbf{1}$ & $\mathrm{n}$ & final \\
\hline 0 & 1 & 1 & 0 & 0 & 1 & 1 & 1 & 2 & $P C=6$ \\
\hline 2 & 2 & 2 & 0 & 1 & $n$ & $n$ & 2 & 2 & $P C=4$ \\
\hline 2 & 2 & 0 & 1 & 1 & 0 & 2 & 2 & 2 & $P C=3$ \\
\hline
\end{tabular}

Table 1: The workings of the noncover $n$-algorithm

By $P C=2$ we mean that at this stage the pending conjunction is the second one, i.e. the one that defines $\operatorname{Mod}_{2}$. In other words, we need to sieve out those $x \in \operatorname{Mod}_{1}$ that happen to be in $\operatorname{Mod}_{2}=(2,2,2, n, n, n, 2,2,2)$. In order to do so we determine the intersection $\{-2,-1,0\} \cap$ $\{-1,0,1\}=\{-1,0\}$ of the " $n$-pools" of $\operatorname{Mod}_{1}$ and $\operatorname{Mod}_{2}$ and then split the $\{0,1,2, n\}$-valued row $r:=\operatorname{Mod}_{1}$ accordingly into a disjoint union $r=r^{\prime} \cup r^{\prime \prime}$ where

$$
\begin{array}{lll}
r^{\prime}:=\left\{x \in r \mid x_{-1}=0 \text { or } x_{0}=0\right\} & =(2,2,2, \mathbf{n}, \mathbf{n}, 2,2,2,2) \\
r^{\prime \prime}:=\left\{x \in r \mid x_{-1}=x_{0}=1\right\} & =(2,2,0, \mathbf{1}, \mathbf{1}, 2,2,2,2) .
\end{array}
$$

While all $x \in r^{\prime}$ trivially satisfy $x_{-1} \wedge x_{0} \wedge x_{1}=0$, i.e. belong to $\operatorname{Mod}_{2}$, this is not the case for all $x \in r^{\prime \prime}$. However, turning at the 6 -th position the 2 to 0 does the job. This yields the 
current working stack with the two rows labelled $P C=3$; see top of Table 1. (Of course this "stack" has nothing to do with its namesake in "stack filter".) As a general rule, the topmost row in the stack is always treated first ("last in, first out"). This may entail "local changes", or a splitting of the top row into several sons. In this way we proceed up to the second last stack in Table 1. Let us pick its top row $r=(2, n, n, 2,0, n, n, n, 2)$ and illustrate once more the splitting process. The intersection of the $n$-pool of $r$ with (the index set of) the pending 6 th conjunction is $\{-3,-2,1,2,3\} \cap\{-3,-2,-1,2,3,4\}=\{-3,-2,2,3\}$. Accordingly split $r$ into the disjoint union of $r^{\prime}$ and $r^{\prime \prime}$ :

$$
\begin{aligned}
& r=(2, n, n, 2,0, n, n, n, 2) \\
& r^{\prime}=(2, \mathbf{n}, \mathbf{n}, 2,0,2, \mathbf{n}, \mathbf{n}, 2) \\
& r^{\prime \prime}=(2, \mathbf{1}, \mathbf{1}, 2,0,0, \mathbf{1}, \mathbf{1}, 2) .
\end{aligned}
$$

Since $r^{\prime} \subseteq \operatorname{Mod}_{6}, r^{\prime}$ is the first son of $r$. We have $r^{\prime \prime} \nsubseteq \operatorname{Mod}_{6}$, but $r^{\prime \prime} \cap \operatorname{Mod}_{6}=(2,1,1, n, 0,0,1,1, n)$ becomes the second son. Both rows are final, i.e. are subsets of Mod and thus collected in a steadily increasing final stack. The working stack now contains three rows with pending conjunctions $6,4,3$ respectively. In our case it just so happens that they are in fact already final (so e.g. all $x$ in the row labelled $P C=4$ happen to satisfy the 4 th, 5 th and 6 th conjunction). The final stack comprises thus the five rows in Table 2 (for the moment ignore $p^{2} q^{2}$ and so forth):

\begin{tabular}{|c|c|c|c|c|c|c|c|c|l}
-4 & -3 & -2 & -1 & 0 & 1 & 2 & 3 & 4 & \\
\hline 2 & 2 & 0 & 1 & 1 & 0 & 2 & 2 & 2 & $p^{2} q^{2}$ \\
\hline 2 & 2 & 2 & 0 & 1 & $n$ & $n$ & 2 & 2 & $p q\left(1-q^{2}\right)=p q-p q^{3}$ \\
\hline 0 & 1 & 1 & 0 & 0 & 1 & 1 & 1 & 2 & $p^{3} q^{5}$ \\
\hline 2 & 1 & 1 & $n$ & 0 & 0 & 1 & 1 & $n$ & $p^{2} q^{4}\left(1-q^{2}\right)=p^{2} q^{4}-p^{2} q^{6}$ \\
\hline 2 & $n$ & $n$ & 2 & 0 & 2 & $n$ & $n$ & 2 & $p\left(1-q^{4}\right)=p-p q^{4}$ \\
\hline
\end{tabular}

Table 2: The probability contributions of the final rows

For instance, the second row in Table 2 contains $2^{5} \cdot\left(2^{2}-1\right)$ noncovers, where $\left(2^{2}-1\right)$ comes from $n n$. The total number $N$ of noncovers evaluates to

$$
N=32+32 \cdot 3+2+2 \cdot 3+16 \cdot 15=376
$$

which is much higher than the number $R=5$ of final multivalued rows. As we shall see in Section 3 , in general the $n$-pool of rows is a bit more subtle.

Let us now calculate the output distribution. The first row in Table 2 contains $2^{5}=32$ bitstrings $x$ with $b_{1}(x)=0$. Each contributes some probability $\alpha_{1} \alpha_{2} p q q p \alpha_{3} \alpha_{4} \alpha_{5}$ to the sum in (2). Since each $\alpha_{i}$ can independently be chosen to be $p$ or $q$, the sum of these 32 terms is

$$
p^{2} q^{2}(p p p p p+\cdots+p q q p q+\cdots+q q q q q)=p^{2} q^{2}(p+q)^{5}=p^{2} q^{2} .
$$

The fact that e.g. $n n=\{00,01,10\}$ yields $p p+p q+q p=1-q^{2}$, explains the contribution $p q\left(1-q^{2}\right)$ of the second row. Similarly for the three other rows. Summing up the terms in Table 2 yields

$$
\begin{aligned}
\phi_{S}(p) & =p^{2} q^{2}+p q-p q^{3}+p^{3} q^{5}+p^{2} q^{4}-p^{2} q^{6}+p-p q^{4} \\
& =7 p^{2}-8 p^{3}-8 p^{4}+25 p^{5}-24 p^{6}+11 p^{7}-2 p^{8}
\end{aligned}
$$




\section{$3 \quad$ Theoretic assessment}

Suppose the constraint $A^{*}=\{3,4\}$ is to be imposed on a row $r=(1,2,1,1)$ in the process of the stack filter $n$-algorithm. Then $r$ needs to be cancelled since no member $X \in r$ satisfies $X \nsupseteq A^{*}$. Fortunately, with some precautions the cancellation of rows can be avoided, which is essential in the Theorem below. Another remark about the proof is in order. Apart from the probabilities coupled to the final $\{0,1,2, n\}$-valued rows, the stack filter $n$-algorithm coincides with the noncover $n$-algorithm of [13], which is a special "homogeneous" case of the Horn $n$ algorithm, which in turn is an instance of some principle of exclusion. Since our special case is somewhat buried by this and the technical machinery of [13], yet admits a comparatively smooth proof from scratch, we give that proof below.

Theorem: Suppose the stack filter $S$ has window size $w$ and its positive Boolean function $b(x)$ is given as a disjunction of $h$ conjunctions (DNF). Then the stack filter $n$-algorithm computes the output distribution of $S$ in time $O\left(N w^{2} h^{2}\right)$. Here $N$ is the number of bitstrings $x$ with $b(x)=0$.

Proof. As in the introductory example, the terms in the DNF of $b(x)$ yield subsets $A_{1}^{*}, \ldots, A_{h}^{*}$ of $W:=[w]$ whose models (= simultaneous noncovers) $Y \subseteq W$ we wish to pack in disjoint $\{0,1,2, n\}$-valued rows. Any (not necessarily final) row $\bar{r}$ is called feasible if $Y \in \bar{r}$ for at least one model $Y$. As opposed to other applications of the princple of exclusion, here feasibility is easily tested. Namely, $\bar{r}$ is feasible if and only if

$$
(\forall 1 \leq i \leq h) \quad A_{i}^{*} \nsubseteq \operatorname{ones}(\bar{r}) .
$$

Initially our "working stack" solely comprises the row $r_{0}=(2,2, \ldots, 2)$ of length $w$ which we identify with the powerset of $W$. Note that $r_{0}$ is feasible since $\emptyset \in r$. Row $r_{0}$ carries the pointer $P C\left(r_{0}\right)=1$, where $P C$ stands for "pending constraint". Generally, the top row $r$ of the working stack is treated as follows. If $P C(r)=j$ (for some $j \in[h]$ ) then the set $A_{j}^{*}$ is "imposed" upon $r$, that is, the set $U$ of all $X \in r$ with $X \nsupseteq A_{j}^{*}$ is represented as a disjoint union of rows $r_{1}, \ldots, r_{s}$ where $s \leq w$. That this is always possible (the "core" claim), and costs $O\left(w^{2}\right)$, will be shown in a moment.

Because $r$ was feasible by induction, at least one of its "candidate" sons $r_{1}, \ldots, r_{s}$ will be as well. Since the feasibility of $\bar{r}=r_{j}$ amounts to the truth of (6), it costs $O(\operatorname{shw})=O\left(h w^{2}\right)$ to sieve the sons of $r$, i.e. the feasible rows among $r_{1}, \ldots, r_{s}$. Altogether the cost of one imposition of a constraint upon a row is $O\left(w^{2}\right)+O\left(h w^{2}\right)=O\left(h w^{2}\right)$.

The $R$ final rows can be viewed as the leaves of a tree with root $(2,2, \ldots, 2)$ that has height $h$; each imposition triggers all sons of some node. Therefore the number of impositions is at most $R h$ (distinct final rows, possibly having some of their forefathers in common). It follows that producing the $R$ final rows costs $O\left(R h \cdot h w^{2}\right)=O\left(N h^{2} w^{2}\right)$ in view of $R \leq N$, by the disjointness of final rows. Calculating (as in (4)) the contributions to $\phi_{S}(p)$ of all final rows, and adding them, costs $O(N w)$, which is swallowed by $O\left(N h^{2} w^{2}\right)$.

It remains to verify the core claim, i.e. that $U:=\left\{X \in r: X \nsupseteq A^{*}\right\}\left(A^{*}:=A_{j}^{*}\right)$ can be represented as promised. 
Case (a): $A^{*} \cap \operatorname{zeros}(r) \neq \emptyset$ or $A^{*}$ wholly contains an $n$-bubble of $r$. Then $U=r$, and so put $r_{s}=r_{1}=r$.

Since $r$ is feasible, $A^{*} \subseteq$ ones $(r)$ is impossible, and so the only remaining possibility is

Case (b): $A^{*} \cap$ zeros $(r)=\emptyset$ and $A^{*}$ does not wholly contain an $n$-bubble of $r$ and $A^{*} \nsubseteq \nsubseteq$ ones $(r)$. This is exactly Case 7 in Section 5 of [13], whose essense we repeat here.

\begin{tabular}{|c|c|c|c|c|c|c|c|c|c|c|c|c|c|c}
1 & 2 & 3 & 4 & 5 & 6 & 7 & 8 & 9 & 10 & 11 & 12 & 13 & 14 & \\
\hline 2 & 2 & $n_{1}$ & $n_{1}$ & $n_{2}$ & $n_{3}$ & $n_{3}$ & $n_{4}$ & $n_{1}$ & $n_{2}$ & $n_{3}$ & $n_{3}$ & $n_{4}$ & $n_{4}$ & $r$ \\
\hline \hline 2 & 2 & $\mathbf{n}$ & $\mathbf{n}$ & $n_{2}$ & $n_{3}$ & $n_{3}$ & $n_{4}$ & 2 & $n_{2}$ & $n_{3}$ & $n_{3}$ & $n_{4}$ & $n_{4}$ & $r_{1}$ \\
\hline 2 & 2 & $\mathbf{1}$ & $\mathbf{1}$ & $\mathbf{0}$ & $n_{3}$ & $n_{3}$ & $n_{4}$ & 0 & 2 & $n_{3}$ & $n_{3}$ & $n_{4}$ & $n_{4}$ & $r_{2}$ \\
\hline 2 & 2 & $\mathbf{1}$ & $\mathbf{1}$ & $\mathbf{1}$ & $\mathbf{n}$ & $\mathbf{n}$ & $n_{4}$ & 0 & 0 & 2 & 2 & $n_{4}$ & $n_{4}$ & $r_{3}$ \\
\hline 2 & 2 & $\mathbf{1}$ & $\mathbf{1}$ & $\mathbf{1}$ & $\mathbf{1}$ & $\mathbf{1}$ & $\mathbf{0}$ & 0 & 0 & $n_{3}$ & $n_{3}$ & 2 & 2 & $r_{4}$ \\
\hline$n$ & $n$ & $\mathbf{1}$ & $\mathbf{1}$ & $\mathbf{1}$ & $\mathbf{1}$ & $\mathbf{1}$ & $\mathbf{1}$ & 0 & 0 & $n_{3}$ & $n_{3}$ & $n_{4}$ & $n_{4}$ & $r_{5}$ \\
\hline \hline
\end{tabular}

Table 3: Five candidate sons of some $\{0,1,2, n\}$-valued row

Suppose $W=[14], A^{*}=[8]$, and $r$ is as in Table 3. Note that the $n$-pool of $r$ is the disjoint union $\{3,4,9\} \cup\{5,10\} \cup\{6,7,11,12\} \cup\{8,13,14\}$ of four mutually independent $n$-bubbles, each one defined by "at least one nul there", as in Section 2. Putting

$r_{1}:=\{X \in r: X \nsupseteq\{3,4\}\}$

$r_{2}:=\{X \in r: X \supseteq\{3,4\}$ and $X \nsupseteq\{5\}\}$

$r_{3}:=\{X \in r: X \supseteq\{3,4,5\}$ and $X \nsupseteq\{6,7\}\}$

$r_{4}:=\{X \in r: X \supseteq\{3,4,5,6,7\}$ and $X \nsupseteq\{8\}\}$

$r_{5}:=\{X \in r: X \supseteq\{3,4,5,6,7,8\}$ and $X \nsupseteq\{1,2\}\}$

it is clear that $U$ is the disjoint union of $r_{1}, \ldots, r_{5}$. A minute's reflection shows that, crucially, these sets can again be written as $\{0,1,2, n\}$-valued rows as shown in Table 3 , and that generally (full details in [13]) splitting a row in $s \leq w$ candidate sons like this costs $O(s w)=O\left(w^{2}\right)$.

It follows from the proof that $O\left(N w^{2} h^{2}\right)$ could be substituted by $O\left(R w^{2} h^{2}\right)$ where $R \leq N$ is the number of final $\{0,1,2, n\}$-valued rows. Unfortunately $R$ is unpredictable. Theoretically $R=N$ is possible but in practise $R$ is usually orders of magnitudes smaller than $N$ (see Subsection $4.1)$.

It has been pointed out that $b(x)$ may not initially be given in disjunctive normal form. However, if not, there are efficient methods to compute the DNF from any reasonable kind of presentation of $b(x)$; this e.g. applies to the erosion - dilation cascades in subsection 4.1. In any case, the bigger problem arguably is to find the bitstrings $x \in\{0,1\}^{w}$ with $b(x)=0$.

\footnotetext{
${ }^{\dagger}$ Even then, provided $N \approx \frac{1}{2} \cdot 2^{W}$ as is to be expected for random PBF's, the stack filter algorithm would beat by a factor 2 a brute force search of all of $\{0,1\}^{W}$.
} 


\section{Related matters}

Subsection 4.1 glimpses at the practical performance of a Mathematica implementation of the stack filter $n$-algorithm, and 4.2 shows that the so called rank selection probabilities $p_{i}$ can be gleaned from the $\{0,1,2, n\}$-valued rows along the way. Subsections 4.3 and 4.4 are about the joint distribution of stack filters, respectively about a certain generalization of "ordinary" stack filters to "balanced" stack filters. The required adaptions of our algorithm stay within the realm of $\{0,1,2, n\}$-valued rows. Finally, as powerful as binary decision diagrams often are, it is argued in 4.5 that they are not appropriate in our situation.

\subsection{Numerics exemplified on the $L U L U$ filter $C_{5}$}

Certain stack filters $L_{n}$, their duals $U_{n}$, and compositions thereof (called $L U L U$ filters) have been proposed in [9] and earlien $\$$, as alternatives to the popular median filters. Actually, the function $b_{1}\left(x_{-4}, \ldots, x_{4}\right)$ from Section 2 is the PBF underlying $U_{2} L_{2}$.

The natural definition of each $L U L U$ filter is as a cascade of so called erosions and dilations (CED), two dual concepts from Mathematical Morphology [9, III.C]. Computing the DNF of any CED essentially amounts $\$$ to calculating CNF's and DNF's of successively bigger (details in [3]) positive Boolean functions. For instance,

$$
C_{n}:=L_{n} U_{n} L_{n-1} U_{n-1} \ldots L_{1} U_{1}
$$

is a CED stack filter with window size $w=2 n^{2}+2 n+1$. Using Berge's algorithm to compute the DNF of $C_{5}$ from its CED-representation took about 46 hours. Calculating the output distribution $\phi_{C_{5}}(p)=p^{5}+7 p^{6}-\cdots+114680 p^{43}+\cdots+p^{53}$ with the stack filter $n$-algorithm took another 12 hours. At least as illuminating as $\phi_{C_{5}}(p)$ are the so called rank selection probabilities that can be calculated along the way as discussed in the next subsection. The underlying PBF of $C_{5}$ had $N=639^{\prime} 173^{\prime} 390^{\prime} 187^{\prime} 370^{\prime} 752$ models, which were packed in a mere $R=179^{\prime} 244$ final rows. More extensive numerical evaluations of similar implementations of the principle of exclusion (and how they compare to say BDD's) are provided in upcoming publications.

Due to the specific regularities of $U_{n} L_{n}$ its DNF has in fact been discovered by other means [9, p.112] and its distribution transfer was computed independent of its DNF in [3]; it equals

$$
\phi_{U_{n} L_{n}}=1-q^{n+1}-n p q^{n+1}-p q^{2 n+2}-\frac{1}{2}(n-1)(n+2) p^{2} q^{2 n+2} .
$$

One verifies that (7) coincides with (5) for $n=2$. Even the distribution transfer of $C_{n}$ can be determined [3], albeit only by an efficient recursive formula as opposed to the closed form in (7). For all $n \leq 5$ the results agreed with the ones obtained with the stack filter $n$-algorithm, which is a strong indication that both methods are correct.

\footnotetext{
${ }^{\ddagger}$ Using terminology of Mathematical Morphology, $L_{n}$ (dually for $U_{n}$ ) is an opening induced by a line segment of length $n+1$, whence the underlying PBF has window size $2 n+1$. As opposed to the median filters, all $L U L U$ filters $S$ are idempotent $(S \circ S=S)$ and even co-idempotent $((i d-S) \circ(i d-S)=i d-S)$.

${ }^{\S}$ Computing the DNF of a PBF from its CNF is a well researched topic [5], which also amounts to get all minimal transversals of a set system. The author used a refinement of the classic "Berge-algorithm" for the task, does not claim that it competes with the cutting edge algorithms for DNF $\leftrightarrow$ CNF, but feels that the stack filter $n$-algorithm is the right approach once the DNF is given.
} 


\subsection{Rank selection probabilities}

Let $S$ be a stack filter. Given a sequence $Z$ of i.i.d. random variables, the so called rank selection probability $p_{i}$ is defined as the probability that a fixed component of the output series $S Z$ is the $i$-th smallest in the sliding window of length $w$. It is known, [8], [2, p.236] that

$$
p_{i}=\frac{A_{w-i}}{\left(\begin{array}{c}
w \\
w-i
\end{array}\right)}-\frac{A_{w-i+1}}{\left(\begin{array}{c}
w \\
w-i+1
\end{array}\right)}
$$

where $A_{i}$ is the number of bitstrings $x$ with $i$ ones and $w-i$ zeros that have $b(x)=0$. The $A_{i}$ 's can be conveniently calculated in tandem with the evaluation of (2). For instance, as the reader can easily verify, the contribution of the last row in Table 2 to $A_{0}$ up to $A_{7}$ is:

$$
\begin{aligned}
& \begin{array}{ll}
A_{0}: & \left(\begin{array}{l}
8 \\
0
\end{array}\right)=1
\end{array} \\
& \begin{array}{ll}
A_{1}: & \left(\begin{array}{l}
8 \\
1
\end{array}\right)=8
\end{array} \\
& \begin{array}{ll}
A_{2}: & \left(\begin{array}{l}
8 \\
2
\end{array}\right)=28
\end{array} \\
& \begin{array}{ll}
A_{3}: & \left(\begin{array}{l}
8 \\
3
\end{array}\right)=56
\end{array} \\
& A_{4}:\left(\begin{array}{l}
4 \\
0
\end{array}\right)\left(\begin{array}{l}
4 \\
4
\end{array}\right)+\left(\begin{array}{l}
4 \\
1
\end{array}\right)\left(\begin{array}{l}
4 \\
3
\end{array}\right)+\left(\begin{array}{l}
4 \\
2
\end{array}\right)\left(\begin{array}{l}
4 \\
2
\end{array}\right)+\left(\begin{array}{l}
4 \\
3
\end{array}\right)\left(\begin{array}{l}
4 \\
1
\end{array}\right)=69 \\
& A_{5}: \quad\left(\begin{array}{l}
4 \\
1
\end{array}\right)\left(\begin{array}{l}
4 \\
4
\end{array}\right)+\left(\begin{array}{l}
4 \\
2
\end{array}\right)\left(\begin{array}{l}
4 \\
3
\end{array}\right)+\left(\begin{array}{l}
4 \\
3
\end{array}\right)\left(\begin{array}{l}
4 \\
2
\end{array}\right)=52 \\
& A_{6}: \quad\left(\begin{array}{l}
4 \\
2
\end{array}\right)\left(\begin{array}{l}
4 \\
4
\end{array}\right)+\left(\begin{array}{l}
4 \\
3
\end{array}\right)\left(\begin{array}{l}
4 \\
3
\end{array}\right)=22 \\
& A_{7}: \quad\left(\begin{array}{l}
4 \\
3
\end{array}\right)\left(\begin{array}{l}
4 \\
4
\end{array}\right)=4 .
\end{aligned}
$$

We mention that in [6] the optimization of stack filters with respect to certain constraints leads to specific desirable values of $A_{1}, \ldots, A_{w}$. Finding a stack filter $S$ that features these values (at least approximately) is however hard. One may hence be led to compile a catalogue of CED's (see 4.1) with corresponding vectors $\left(A_{1}, \ldots, A_{w}\right)$ from which a suitable candidate $S$ can be picked.

\subsection{The joint output distribution of two stack filters}

Let $Z$ be a doubly infinite sequence of i.i.d. random variables. For two stack filters $S$ and $T$ with corresponding positive Boolean functions $b_{1}(x)$ and $b_{2}(y)$ their joint output distribution $F_{S Z, T Z}(s, t)$, or simply $J D(s, t)$, is defined as

$$
J D(s, t) \quad:=\operatorname{Prob}\left((S Z)_{0} \leq s \text { and }(T Z)_{0} \leq t\right) .
$$

If we set $p:=\operatorname{Prob}\left(Z_{0} \leq s\right), \pi:=\operatorname{Prob}\left(Z_{0} \leq t\right)$ and assume $p \leq \pi$ (the case $p>\pi$ is similar) then it is shown in $[2, \mathrm{p} .230]$ that

$$
J D(s, t)=\sum_{i=0}^{w} \sum_{j=0}^{w} A_{i, j} p^{i}(\pi-p)^{w-i-j}(1-\pi)^{j},
$$


where $A_{i j}$ is the number of $(x, y) \in\{0,1\}^{w} \times\{0,1\}^{w}$ such that

$$
x \geq y, \quad b_{1}(x)=b_{2}(y)=0, \quad v_{-,-}(x, y)=i, \quad v_{+,+}(x, y)=j,
$$

and where

$$
\begin{aligned}
v_{-,-}\left(x_{1}, \ldots, x_{w}, y_{1}, \ldots, y_{w}\right):=\left|\left\{1 \leq k \leq w: x_{k}=y_{k}=0\right\}\right| \\
v_{+,+}\left(x_{1}, \ldots, x_{w}, y_{1}, \ldots, y_{w}\right):=\left|\left\{1 \leq k \leq w: x_{k}=y_{k}=1\right\}\right|
\end{aligned}
$$

The calculation of the coefficients $A_{i j}$ works row-wise. So suppose $r$ in Table 4 is one of the final rows obtained after applying the noncover $n$-algorithm to $b_{1}$. Obviously the set

$$
\mathcal{F}:=\{y:(\exists x \in r) \quad x \geq y\}
$$

is represented by row $r_{0}$. If say $b_{2}(y)=y_{3} \wedge y_{9} \wedge y_{10}$ then the set

$$
\mathcal{F}\left(b_{2}\right):=\left\{y \in \mathcal{F}: b_{2}(y)=0\right\}
$$

is the disjoint union $\rho_{1} \cup \rho_{2} \cup \rho_{3}$ :

\begin{tabular}{c|c|c|c|c|c|c|c|c|c|c|c|} 
& 1 & 2 & 3 & 4 & 5 & 6 & 7 & 8 & 9 & 10 & 11 \\
\hline$r=$ & $n_{1}$ & $n_{1}$ & $n_{1}$ & 2 & 2 & 0 & $n_{2}$ & $n_{2}$ & $n_{2}$ & 1 & 1 \\
\hline$r_{0}=$ & $n_{1}$ & $n_{1}$ & $n_{1}$ & 2 & 2 & 0 & $n_{2}$ & $n_{2}$ & $n_{2}$ & 2 & 2 \\
\hline$\rho_{1}=$ & 2 & 2 & $\mathbf{0}$ & 2 & 2 & 0 & $n_{2}$ & $n_{2}$ & $\mathbf{n}_{\mathbf{2}}$ & $\mathbf{2}$ & 2 \\
\hline$\rho_{2}=$ & $n_{1}$ & $n_{1}$ & $\mathbf{1}$ & 2 & 2 & 0 & 2 & 2 & $\mathbf{0}$ & $\mathbf{2}$ & 2 \\
\hline$\rho_{3}=$ & $n_{1}$ & $n_{1}$ & $\mathbf{1}$ & 2 & 2 & 0 & $n_{2}$ & $n_{2}$ & $\mathbf{1}$ & $\mathbf{0}$ & 2 \\
\hline$x=$ & 0 & 1 & 1 & 1 & 0 & 0 & 1 & 1 & 0 & 1 & 1 \\
\hline$\sigma=$ & 0 & 2 & 2 & 2 & 0 & 0 & $\mathbf{0}$ & $\mathbf{2}$ & 0 & 0 & 2 \\
\hline$\tau=$ & 0 & 2 & 2 & 2 & 0 & 0 & $\mathbf{1}$ & $\mathbf{0}$ & 0 & 0 & 2 \\
\hline
\end{tabular}

Table 4: Adapting the algorithm to joint output distributions

For each $x \in r$ and $k \in\{1,2,3\}$ one now records $v_{-,-}(x, y)$ and $v_{+,+}(x, y)$ for all $y \in \rho_{k}$ with $y \leq x$. For instance, taking the $x$ indicated in Table 4 one verifies that

$$
\left\{y \in \rho_{3}: y \leq x\right\} \quad=\quad \sigma \cup \tau,
$$

where the later union is disjoint (see $n_{2} n_{2}$ in $\rho_{3}$ and the corresponding boldface entries in $\sigma, \tau$ ). It is easy to see that $\sigma$ contributes an amount of $\left(\begin{array}{l}5 \\ j\end{array}\right)$ to the value of $A_{4, j}$ for all $0 \leq j \leq 5$. Similarly $\tau$ contributes an amount of $\left(\begin{array}{l}4 \\ j\end{array}\right)$ to the value of $A_{4, j+1}(0 \leq j \leq 4)$. Calculations can be sped up by clumping together suitable $x$ 's rather than processing them one by one. We discuss a similar phenomenon in more detail in the next subsection.

\subsection{Balanced stack filters}

In [11], [12] the concept of a balanced stack filter $S$ is introduced. Citing from [11]: "They are much more versatile, being empowered not only with lowpass filtering characteristics, but with

IThis notation is not used in [2] but ties in well with the notation used in subsection 4.4, which in turn is akin to the notation of [11]. For instance, our $v_{-,+}(x, y)$ in 4.4 corresponds to $w(\bar{x} \wedge s)$ in equation (17) of [11].

"Actually, Arce, Paredes and Shmulevich propose to reserve the term "stack filter" to their new concept, and to relabel the "old" stack filters as stack smoothers. As suggested by one referee, we stick to the old, well established terminology. 
bandpass or highpass filtering characteristics as well." They are based on "mirrored thresholding" which entails $t$ and $-t$ to play symmetric roles. Most important for us, $S$ is based again upon a PBF albeit in a manner more sophisticated than (1). For instance, the PBF is of the kind $b(x, y)=b\left(x_{1}, \ldots, x_{w}, y_{1}, \ldots, y_{w}\right)$, and in this set up a stack filter turns out to be a balanced stack filter where $b$ does not depend on $y_{1}, \ldots, y_{w}$ (i.e., these variables are fictitious). As usual let $Z$ be a doubly inifinite sequence of i.i.d. random variables with common cumulative distribution function $F_{Z}(t)=\operatorname{Prob}\left(Z_{i} \leq t\right)(i \in \mathbb{Z})$. Put $F(t)=F_{Z}(t)$ and

$$
\begin{aligned}
& p_{+,+}:= \begin{cases}F(-t)-F(t) & \text { if } t \leq 0 \\
0 & \text { if } t>0\end{cases} \\
& p_{-,-}:= \begin{cases}0 & \text { if } t \leq 0 \\
F(t)-F(-t) & \text { if } t>0\end{cases} \\
& p_{-,+}:= \begin{cases}F(t) & \text { if } t \leq 0 \\
F(-t) & \text { if } t>0\end{cases} \\
& p_{+,-}:= \begin{cases}1-F(-t) & \text { if } t \leq 0 \\
1-F(t) & \text { if } t>0 .\end{cases}
\end{aligned}
$$

Besides $v_{+,+}(x, y)$ and $v_{-,-}(x, y)$ from 4.3 we also put

$$
\begin{aligned}
& v_{-,+}(x, y):=\mid\left\{1 \leq k \leq w: x_{k}=0 \text { and } y_{k}=1\right\} \mid \\
& v_{+,-}(x, y):=\mid\left\{1 \leq k \leq w: x_{k}=1 \text { and } y_{k}=0\right\} \mid .
\end{aligned}
$$

Modulo some obvious typos, it is shown in $[11,(17)]$ that the output distribution, i.e. $F_{S Z}(t)=$ $\operatorname{Prob}\left((S Z)_{0} \leq t\right)$, can be calculated as

$$
F_{S Z}(t)=\sum_{b(x, y)=0} p_{+,+}^{v_{+,+}(x, y)} \cdot p_{+,-}^{v_{+,-}(x, y)} \cdot p_{-,+}^{v_{-,+}(x, y)} \cdot p_{-,-}^{v_{-,-}(x, y)} .
$$

As opposed to $J D(s, t)$ in (8), which is a polynomial of $\operatorname{Prob}\left(Z_{0} \leq s\right)$ and $\operatorname{Prob}\left(Z_{0} \leq t\right)$, here $F_{S Z}(t)$ is not quite a polynomial in terms of $\operatorname{Prob}\left((S Z)_{0} \leq t\right)$ and $\operatorname{Prob}\left((S Z)_{0} \leq-t\right)$.

Nevertheless the noncover $n$-algorithm is of good use. Suppose it has (among others) returned the final row $r$ in Table 5. Take any bitstring $x^{*}=\left(x_{1}, \ldots, x_{9}\right)$ "contained" in the left hand side $\left(n_{1}, n_{2}, n_{3}, 1, n_{4}, n_{4}, 0,2, n_{3}\right)$ of $r$. More precisely, any bitstring $x^{*}$ which is extendible** to a bitstring $\left(X^{*}, y\right) \in r$. Say $x^{*}=(1,1,1,1,1,0,0,0,0)$. For each fixed $k \in\{0,1, \ldots, 5\}$ and $k^{\prime} \in\{0,1, \cdots, 4\}$ we now show how the number $f\left(k, k^{\prime}\right)$ of bitstrings $y=\left(y_{1}, \ldots, y_{9}\right)$ with

$$
\begin{gathered}
v_{+,+}\left(x^{*}, y\right)=k \text { and } v_{-,+}\left(x^{*}, y\right)=k^{\prime} \\
\text { (whence } \left.v_{+,-}\left(x^{*}, y\right)=5-k \text { and } v_{-,-}\left(x^{*}, y\right)=4-k^{\prime}\right)
\end{gathered}
$$

can be calculated fast. First, notice that the subset

$$
r\left(x^{*}\right):=\quad\left\{(x, y) \in r: x=x^{*}\right\}
$$

of $r$ can be written as multi-valued row as shown in Table 5.

\footnotetext{
${ }^{* *}$ It is easily seen that the extendible bitstrings are exactly the members of $\left(2,2,2,1, n_{4}, n_{4}, 0,2,2\right)$.
} 


\begin{tabular}{|c|c|c|c|c|c|c|c|c|c|c|c|c|c|c|c|c|c|c|}
\hline & $x_{1}$ & $x_{2}$ & $x_{3}$ & $x_{4}$ & $x_{5}$ & $x_{6}$ & $x_{7}$ & $x_{8}$ & $x_{9}$ & $y_{1}$ & $y_{2}$ & $y_{3}$ & $y_{4}$ & $y_{5}$ & $y_{6}$ & $y_{7}$ & $y_{8}$ & $y_{9}$ \\
\hline & & & & & & & & & & & & & & & & & & \\
\hline$r=$ & $n_{1}$ & $n_{2}$ & $n_{3}$ & 1 & $n_{4}$ & $n_{4}$ & 0 & 2 & $n_{3}$ & $n_{1}$ & $n_{1}$ & $n_{3}$ & $n_{2}$ & $n_{2}$ & $n_{1}$ & $n_{1}$ & $n_{2}$ & $n_{2}$ \\
\hline$r\left(x^{*}\right)=$ & 1 & 1 & 1 & 1 & 1 & 0 & 0 & 0 & 0 & $n_{1}$ & $n_{1}$ & 2 & $n_{2}$ & $n_{2}$ & $n_{1}$ & $n_{1}$ & $n_{2}$ & $n_{2}$ \\
\hline & & & & & & & & & & & & & & & & & & \\
\hline$r_{1}=$ & 1 & 1 & 1 & 1 & 1 & 0 & 0 & 0 & 0 & $\mathbf{n}_{1}$ & $\mathbf{n}_{1}$ & 2 & $\mathbf{n}_{2}$ & $\mathbf{n}_{2}$ & 2 & 2 & 2 & 2 \\
\hline$r_{2}=$ & 1 & 1 & 1 & 1 & 1 & 0 & 0 & 0 & 0 & $\mathbf{n}_{1}$ & $\mathbf{n}_{1}$ & 2 & 1 & 1 & 2 & 2 & $n_{2}$ & $n_{2}$ \\
\hline$r_{3}=$ & 1 & 1 & 1 & 1 & 1 & 0 & 0 & 0 & 0 & 1 & 1 & 2 & $\mathbf{n}_{2}$ & $\mathbf{n}_{2}$ & $n_{1}$ & $n_{1}$ & 2 & 2 \\
\hline$r_{4}=$ & 1 & 1 & 1 & 1 & 1 & 0 & 0 & 0 & 0 & 1 & 1 & 2 & 1 & 1 & $n_{1}$ & $n_{1}$ & $n_{2}$ & $n_{2}$ \\
\hline
\end{tabular}

Table 5: Adapting the algorithm to balanced stack filters

Problem is we cannot freely choose $k 1$ 's among $\left\{y_{1}, \ldots, y_{5}\right\}$ and $k^{\prime} 1$ 's among $\left\{y_{6}, \ldots, y_{9}\right\}$ because e.g. the choice $(1,1,0,0,0,1,1,0,0)$ clashes with $n_{1} n_{1} n_{1} n_{1}$. But when one partitions $r\left(x^{*}\right)$ as $r_{1} \cup r_{2} \cup r_{3} \cup r_{4}$ as indicated, then for each $r_{i}$ the choices within $\left\{y_{1}, \ldots, y_{5}\right\}$ respectively $\left\{y_{6}, \ldots, y_{9}\right\}$ can be made independently. To fix ideas, say $k=2$ and $k^{\prime}=3$. Then the contribution of $r\left(x^{*}\right)=r_{1} \cup r_{2} \cup r_{3} \cup r_{4}$ to the coefficient of the monom

$$
p_{+,+}^{k} p_{+,-}^{5-k} p_{-,+}^{k^{\prime}} p_{-,-}^{4-k^{\prime}}
$$

occuring in $F_{S X}(t)$ is

$$
f\left(k, k^{\prime}\right)=8 \cdot 4+1 \cdot 2+1 \cdot 2+0 \cdot 0=36 .
$$

Generally, the number of bitstrings with a fixed number $k$ of 1 's that are contained in a $\{0,1,2, n\}$-valued row can be determined fast. Similar to 4.3 , but more obvious, time can be saved by clumping together suitable bitstrings $\left(x_{1}, \ldots, x_{9}\right)$. For instance, $(1,1,0,1,0,0,0,1,1)$ causes the same right hand side $\left(n_{1}, n_{1}, 2, n_{2}, n_{2}, n_{1}, n_{1}, n_{2}, n_{2}\right)$ as did $x^{*}$. As another example, $(0,0,1,1,1,0,0,0,0)$ is one among ten left hand sides of weight 3 that cause the right hand side $(2,2,2,2,2,2,2,2,2)$.

\subsection{On binary decision diagrams}

Shmulevich et al. [10] proposed to evaluate (2) by setting up a binary decision diagram (BDD) for the Boolean function $b(x)$ that underlies the stack filter $S$ whose distribution transfer needs to be calculated. Suppose one has indeed spent time to get a BDD that represents $b(x)$. While the number of models $x \in\{0,1\}^{w}$ with $b(x)=0$ can be determined fast from a BDD, it is more cumbersome to generate all models, as is forced by (2). True, from the BDD one can get the set of models as a disjoint union of $\{0,1,2\}$-valued rows in recursive fashion. (See [1, p.22] or the long chapter on BDDs in Donald Knuth's forthcoming book.) However, these rows are far more numerous than the ones produced by the stack filter $n$-algorithm; not surprisingly since our algorithm uses one additional symbol and hence more flexibililty in its $\{0,1,2, n\}$-valued rows. Finally, the enhancements discussed in subsections 4.2, 4.3, 4.4 are cumbersome to be handled by BDD's. 


\section{Conclusion}

The present article can be viewed as the realization of a fifth benefit of DNF's that was announced in [14], i.e. the calculation of a stack filter's output distribution and (even more useful) its selection probabilities. The so doing stack filter $n$-algorithm is accessible from the author's home page. It has the form of a Mathematica Notebook. The indicated enhancements in 4.3 and 4.4 have not been programmed by the author; anybody is welcome to do so.

Last not least we draw attention to [7], a comprehensive framework in which stack filters, alias lattice polynomial functions (LPF), constitute but one type of aggregation function. However, there are no references to nonlinear signal theory or Mathematical Morphology in [7]. For instance, other than might appear from [7, p.361], cumulative distribution functions of "nice" LPF's (i.e. their underlying PBF's are more regular than ours) have a long history - in the case of Order Statistics dating back to 1932 [4].

\section{References}

[1] H.R. Andersen, An introduction to Binary Decision Diagrams, lecture notes, IT University of Copenhagen, 1999.

[2] J. Astola, P. Kuosmanen, Fundamentals of nonlinear digital filtering, CRC Press 1997.

[3] R. Anguelov, P.W. Butler, C.H. Rohwer, M. Wild, Calculating the output distribution of stack filters that are erosion-dilation cascades, in particular $L U L U$-filters, submitted.

[4] A.T. Craig, On the distribution of certain statistics, Amer. J. Math. 54 (1932) 353-366.

[5] T. Eiter, K. Makino, G. Gottlob, Computational aspects of monotone dualization: A brief survey, Disc. Appl. Math. 156 (2008) 2035-2049.

[6] P. Kuosmanen, J. Astola, Optimal stack filters under rank selection and structural constraints, Signal Processing 41 (1995) 309-338.

[7] M. Grabisch, J.L. Marichal, R. Mesiar, E. Pap, Aggregation functions, Encyclopedia of Mathematics and its Applications 127, Cambridge University Press 2009.

[8] P. Kuosmanen, J. Astola, S. Agaian, On rank selection probabilities, IEEE Trans. on Signal Proc. 42 (1994) 3255-3258.

[9] C. Rohwer, M. Wild, LULU Theory, idempotent stack filters, and the mathematics of vision of Marr, Advances in Imaging and Electron Physics 146 (2007) 57-162.

[10] I. Shmulevich, K. Egiazarian, O. Yli-Harja, J. Astola, Efficient computation of output distributions of stack filters using ordered binary decision diagrams, Journal of Signal Processing 4 (2000) 195-200.

[11] I. Shmulevich, J.L. Paredes, G.R. Arce, Output distributions of stack filters based on mirrored threshold decomposition, IEEE Transactions on Signal Processing 49 (2001) 14541460 . 
[12] J.L. Paredes, G.R. Arce, Stack filters, stack smoothers, and mirrored threshold decomposition, IEEE Transactions on signal processing, Volume 47, Issue: 10, pp.2757-2767, Oct 1999.

[13] M. Wild, Compactly generating all satisfying truth assignments of a Horn formula, Journal on Satisfiability, Boolean Modeling and Computation 8 (2012) 63-82.

[14] M. Wild, The many benefits of putting stack filters in disjunctive or conjunctive normal form, Disc. Appl. Math. 149 (2005) 174-191.

[15] O. Yli-Harja, J. Astola, Y. Neuvo, Analysis of the properties of median and weighted median filters using threshold logic and stack filter representation, IEEE Trans. Signal Proc. 39 (1991) 395-410. 